\title{
Ultracold Few-Boson Systems in a Double-Well Trap
}

\author{
Sascha Zöllner|* \\ Theoretische Chemie, Institut für Physikalische Chemie, \\ Universität Heidelberg, INF 229, 69120 Heidelberg, Germany \\ Hans-Dieter Meyen $\dagger$ \\ Theoretische Chemie, Institut für Physikalische Chemie, \\ Universität Heidelberg, INF 229, 69120 Heidelberg, Germany \\ Peter Schmelcher \\ Theoretische Chemie, Institut für Physikalische Chemie, \\ Universität Heidelberg, INF 229, 69120 Heidelberg, Germany and \\ Physikalisches Institut, Universität Heidelberg, \\ Philosophenweg 12, 69120 Heidelberg, Germany
}

(Dated: 5/24/2006)

\begin{abstract}
We investigate the transition of a quasi-one-dimensional few-boson system from a weakly correlated to a fragmented and finally a fermionized ground state. Our numerically exact analysis, based on a multi-configurational method, explores the interplay between different shapes of external and inter-particle forces. Specifically, we demonstrate that the addition of a central barrier to an otherwise harmonic trap may supports the system's fragmentation, with a symmetry-induced distinction between even and odd atom numbers. Moreover, the impact of inhomogeneous interactions is studied, where the effective coupling strength is spatially modulated. It is laid out how the ground state can be displaced in a controlled way depending on the trap and the degree of modulation. We present the one- and two-body densities and, beyond that, highlight the role of correlations on the basis of the natural occupations.
\end{abstract}

PACS numbers: 03.75.Hh, 03.65.Ge, 03.75.Nt

*Electronic address: sascha@pci.uni-heidelberg.de

$\dagger$ Electronic address: dieter@pci.uni-heidelberg.de 
‡Electronic address: peter@pci.uni-heidelberg.de 


\section{INTRODUCTION}

Ever since the first realization of a Bose-Einstein condensate, trapped ultracold atoms have been the focal point of an enormous number of research efforts, both from the experimental and the theoretical side [1, 2, 3, 4]. Allowing for an undreamt-of level of control, regarding the adjustment of the external as well as the inter-particle forces via electromagnetic fields, they may serve as some kind of Rosetta stone for various research areas, ranging from solid-state physics to optics.

A special focus rests on the aspect of a system's dimensionality. Particles restricted to a lower-dimensional subspace, such as a wave guide in the one-dimensional case, often unveil features that are conspicuously different from isotropic ones. A striking example is the scattering theory of an ultracold system whose transverse degrees of freedom are frozen such that an effective one-dimensional description becomes possible, as developed by Olshanii [5]. In that case, the effective interaction strength of the system may be tuned at will from a weakly correlated to a strongly repulsive system to an attractive one by merely changing the lengthscale of the transverse confinement. A particularly absorbing issue is the so-called Tonks-Girardeau gas of impenetrable bosons [6], which provides an analogy to an ideal gas of fermions and has recently been realized experimentally [7, 8].

Traditionally, the physics of ultracold atoms has been studied extensively on the premise of large numbers of atoms $N$ and sufficiently small interaction. This legitimates the use of the Gross-Pitaevskii equation [1], which rests on the assumption of a macroscopic wave function composed of a single orbital. An extension of this idea to more than one orbital, the so-called best mean field [9], has indeed proven to be very efficient in giving a qualitative picture of the pathway from condensates to fragmentation, which occurs when the interactions become strong enough to deplete the single-orbital 'condensate' [10].

Still, there are several rationales to consider systems of few atoms, typically $N \sim 1-$ 100. For one thing, the interesting situation of a strongly correlated gas is experimentally accessible only for small systems. Yet a more fundamental argument is that few-particle systems permit a much higher level of control. There is no thermal cloud, as for large $N$, associated with decoherence and energetically dense manifolds of excitations, but a pure quantum system. Instead, finite-size effects become relevant, and two-body correlations have to be taken into account from the start. Conversely, few-body systems are indeed amenable 
to ab-initio calculations, making it tempting to analyze their features in detail and without resort to uncontrolled approximations.

While the last statement is true in principle, it is generally highly challenging from a computational standpoint. In fact, many attempts to study few-body systems are based either on analytic solutions for simple model systems [11, 12, 13, 14, 15] or are restricted to very few atoms [16, 17, 18, 19]. Moreover, some numerically exact methods have been put forward recently. Part of these are actually designed for larger systems but include only few orbitals as in double-well traps $|20,21|$. while others regard generic model systems such as the simple harmonic oscillator [22, 23, 24] or a double well [25].

The goal of this paper is to investigate the interplay between external forces, on the one hand, and the effect of manipulating the inter-particle forces. Our investigation focuses on the numerically exact ground state obtained via the Multi-Configuration Time-Dependent Hartree method [26, 27, 28]. We consider the example of $N=2, \ldots, 6$ one-dimensional repulsive bosons in a double well, whose barrier separating both wells can be adjusted so that both a purely harmonic trap as well as large barriers are accessible. We analyze how this competes with the effect of an increasing interaction strength, which leads to fragmentation and finally fermionization of the ensemble. That interplay is taken one step further by considering a setup where the interaction is inhomogeneous, i.e., the inter-particle forces depend on the position of a collision, too. This may prove to be a valuable tool on the road to extracting single atoms from an ensemble in a controlled way [29, 30]. If the interaction strength is slightly higher on one side of the trap, the ground state can be shown to be displaced to the other side. The nature of this displacement and its dependency on the trap configuration as well as the interaction's strength and modulation are studied.

This article is organized as follows. In Sec. III, the model is introduced and the relevant parameter regimes are discussed. Sec. III contains a concise introduction to the computational method and how it can be applied to our problem. In the subsequent section the few-boson system is studied for standard, homogeneous interactions, casting light on the passage between the low- and strong-correlation regime, and what effect the trap configuration has. The one- and two-particle densities are displayed in Secs. IVA/IVB, while the deeper role of fragmentation is highlighted in IVD. The same program is carried out for the case of a collisionally inhomogeneous setup in Sec. V 


\section{THE MODEL}

In this article we investigate a system of few interacting bosons $(N=2, \ldots, 6)$ in an external trap. These particles, typically atoms with mass $M$, are taken to be one-dimensional (1D) - more precisely, we assume the other two degrees of freedom to be frozen out in a sense described below.

Let the original 3D system be modeled by the Hamiltonian

$$
H=\sum_{i} h_{i}+\sum_{i<j} V\left(\mathbf{r}_{i}-\mathbf{r}_{j}\right)
$$

where $h=\frac{1}{2 M} \mathbf{p}^{2}+U(\mathbf{r})$ is the one-particle (1P) Hamiltonian with a trapping potential $U$, while $V$ is the two-particle interaction potential with certain low-energy scattering parameters $a_{0}, r_{0}$ (scattering length and effective range, respectively). It is well-known that for sufficiently low momenta $\left(k r_{0} \ll 1\right)$ the details of the interaction become irrelevant. More precisely, if any other interaction potential is taken, the energy as well as the asymptotic wave function will remain unchanged as long as $a_{0}$ agrees (and $r_{0}$, in the next order). In particular, it is usually convenient to model $V$ by an effective point interaction, the so-called regularized pseudo-potential [31]

$$
V(\mathbf{r})=\frac{4 \pi a_{0}}{M} \delta(\mathbf{r}) \partial_{r} r
$$

\section{A. Effective 1D Hamiltonian}

We are now interested in the quasi-1D case, where the trap is supposed to be anisotropic such that there is a 'transversal' direction $(\perp)$ with a characteristic length $a_{\perp}$ much smaller than that of the longitudinal direction, $a_{\|}$. In other words, the transverse energy gap be sufficiently large compared to the accessible energy of the system, so that ( $\|)$ may be regarded as virtually the only degree of freedom. In this case, one may integrate out the 'frozen' transversal subsystem so as to obtain an effective 1D interaction [5]

$$
V(x)=g_{1 \mathrm{D}} \delta(x), \text { with } g_{1 \mathrm{D}}=\frac{4 a_{0}}{M a_{\perp}^{2}}\left(1-\mathcal{C} \frac{a_{0}}{a_{\perp}}\right)^{-1} \quad(\mathcal{C}=1.4603 \ldots) .
$$

(These results were derived on the premise of a harmonic transverse trap potential, that is, $a_{\perp}=1 / \sqrt{M \omega_{\perp}}$.) It is this effective interaction that shall serve as the base for our 
investigations, yet with two qualifications. Firstly, the homogeneity of $V$ will be abandoned later in Sec. $\mathrm{V}$, where that modification is discussed in detail. Secondly, though this delta-type potential is convenient and yields an immediate connection to the experimentally relevant parameters, it is notoriously intractable in ab-initio computations like ours. This is a fundamental fact: By construction, the pseudopotential imposes the condition $\psi^{\prime}\left(0^{+}\right)-\psi^{\prime}\left(0^{-}\right)=2 g_{1 \mathrm{D}} \psi(0)$ on the derivative of the relative coordinate-i.e., whenever two

particles meet, the wave function behaves like $e^{-\kappa|x|}$. This non-smoothness is of course unphysical and solely serves to impose the correct asymptotics on the wave function. In an exact calculation, when the problem is approximated by $C^{\infty}$-functions, this leads to convergence problems. It would be much less artificial to use a more realistic interaction with a non-zero effective range as a remedy. We thus opt to mollify the delta function, and instead use $V(x)=g_{1 \mathrm{D}} \delta_{\sigma}(x)$ with the normalized Gaussian

$$
\delta_{\sigma}(x)=\frac{\exp \left(-\frac{x^{2}}{2 \sigma^{2}}\right)}{\sqrt{2 \pi} \sigma}
$$

which tends to $\delta$ as $\sigma \rightarrow 0$ in the distribution sense. In both analytic and numerical model calculations, we ascertained that for $\sigma \ll 1 /\left|g_{1 \mathrm{D}}\right|$ the results are actually quite close to the limit $\sigma \rightarrow 0$. On the other hand, the width $\sigma$ should not be too small so as to accurately sample the Gaussian. As a trade-off, we choose a fixed value $\sigma / a_{\|}=.05$. In principle, computations for more than one width could be done in order to extrapolate to zero; however, we will always keep it fixed.

\section{B. Scaling}

For reasons of universality as well as computational aspects, we will work with a Hamiltonian rescaled to the lengthscale of the 1D-longitudinal system, $a_{\|}$. More specifically, we carry out a global coordinate transform $Q^{\prime}:=Q / a_{\|}$, with $Q \equiv\left(x_{1}, \ldots, x_{N}\right)^{T}$, which leads to

$$
\underbrace{H(Q) / \omega_{\|}}_{=: H^{\prime}\left(Q^{\prime}\right)}=\sum_{i}\left(-\frac{1}{2} \partial_{i}^{\prime 2}+U^{\prime}\left(x_{i}^{\prime}\right)\right)+\sum_{i<j} V^{\prime}\left(x_{i}^{\prime}-x_{j}^{\prime}\right) .
$$

Here $\omega_{\|} \equiv 1 / M a_{\|}^{2}$ defines the energy scale, and $U^{\prime}\left(x^{\prime}\right):=U\left(x=x^{\prime} a_{\|}\right) / \omega_{\|}$etc. denotes the rescaled potential deprived of any dimensionful parameters. $H^{\prime}$ naturally lends itself as a convenient working Hamiltonian, and we will skip any primes in the following sections. 
As an illustration, the $1 \mathrm{D}$ point interaction reduces to

$$
V^{\prime}\left(x^{\prime}\right)=g_{1 \mathrm{D}}^{\prime} \delta\left(x^{\prime}\right), \quad g_{1 \mathrm{D}}^{\prime}:=\frac{4 a_{0}^{\prime}}{a_{\perp}^{\prime 2}}\left(1-\mathcal{C} \frac{a_{0}^{\prime}}{a_{\perp}^{\prime}}\right)^{-1} .
$$

The only relevant parameter of the interaction is thus the scaled interaction strength, which in turn requires only the knowledge of the (scaled) scattering length $a_{0}^{\prime}=a_{0} / a_{\|}$and the transverse dimension $a_{\perp}^{\prime}=a_{\perp} / a_{\|}$.

\section{Parameter regimes}

As mentioned above, two parameters enter our Hamiltonian: $a_{0}^{\prime}=a_{0} / a_{\|}$and $a_{\perp}^{\prime}=a_{\perp} / a_{\|}$. Both of course depend on

- the $1 \mathrm{D}$ length scale $a_{\|}=1 / \sqrt{M \omega_{\|}}$(due to scaling)

- the scattering length $a_{0}<a_{\|}$of the atomic species considered (about 100 a.u. for alkalis; only positive values are considered here).

- the transversal lengthscale $a_{\perp} \ll a_{\|}$. Of course $a_{\perp}>a_{0}$ is required unless the validity of the 'bare' pseudopotential is put into question. We put $a_{\perp}=0.1 a_{\|}$for simplicity.

According to (11), $g_{1 D}$ does not depend linearly on $a_{0}$, but rather tends to $+\infty$ as $a_{0} \rightarrow a_{\perp} / \mathcal{C}$ from below. In other words, the system becomes strongly correlated when the scattering length approaches the transverse-confinement scale, no matter if the 3D system was strongly interacting to begin with. We restrict our attention to $g \equiv g_{1 \mathrm{D}}>0$. Table \illustrates the range of values of $a_{0}^{\prime}$ for different (longitudinal) trap frequencies $\omega_{\|}$, and what $g_{1 \mathrm{D}}^{\prime}$ they correspond to for $\mathrm{Na} / \mathrm{Rb}$ (at fixed $a_{\perp}^{\prime}=.1$ ).

\section{COMPUTATIONAL METHOD}

Our goal is to investigate the ground state of the system introduced in Sec. II for all relevant interaction strengths in a numerically exact fashion. In other words, our approach is not to approximate the problem by resorting to two-mode or mean-field descriptions, but rather to approach the solution in a controllable way. It has to be stressed that this is a highly 


\begin{tabular}{|c|c|c||c|c|}
\hline$\omega_{\|} / 2 \pi \mathrm{Hz}$ & $a_{0}^{\prime}(\mathrm{Na})$ & $g_{1 \mathrm{D}}^{\prime}$ & $a_{0}^{\prime}(\mathrm{Rb})$ & $g_{1 \mathrm{D}}^{\prime}$ \\
\hline \hline $10^{2}$ & $1.9 \cdot 10^{-3}$ & .78 & $5 \cdot 10^{-3}$ & 2.2 \\
\hline $10^{3}$ & $6 \cdot 10^{-3}$ & 2.6 & $1.6 \cdot 10^{-2}$ & 8.3 \\
\hline $10^{4}$ & $1.9 \cdot 10^{-2}$ & 105 & $5 \cdot 10^{-2}$ & 77 \\
\hline $10^{5}$ & $6 \cdot 10^{-2}$ & 189 & $1.6 \cdot 10^{-1}$ & -48 \\
\hline
\end{tabular}

Table I: Values of the scaled coupling strength $g_{1 \mathrm{D}}^{\prime}$ for Sodium and Rubidium for different trap frequencies $\omega_{\|} / 2 \pi$ and $a_{\perp}^{\prime}=.1$.

challenging and time-consuming task, and only few such studies on ultracold atoms exist even for model systems [20, 21, 22]. Our approach relies on the Multi-Configuration TimeDependent Hartree (MCTDH) method [26, 28, 32], primarily a wave-packet dynamics code known for its outstanding efficiency in high-dimensional applications. To be self-contained, a concise introduction to this tool - and how it can be adapted to our purposes - is presented in this section.

The underlying idea of MCTDH is to solve the time-dependent Schrödinger equation

$$
\left\{\begin{array}{c}
i \dot{\Psi}=H \Psi \\
\Psi(Q, 0)=\Psi_{0}(Q)
\end{array}\right.
$$

as an initial-value problem by expansion in terms of direct (or Hartree) products $\Phi_{J}$ :

$$
\Psi(Q, t)=\sum_{J} A_{J}(t) \Phi_{J}(Q, t) \equiv \sum_{j_{1}=1}^{n_{1}} \ldots \sum_{j_{f}=1}^{n_{f}} A_{j_{1} \ldots j_{f}}(t) \prod_{\kappa=1}^{f} \varphi_{j_{\kappa}}^{(\kappa)}\left(x_{\kappa}, t\right)
$$

using a convenient multi-index notation for the configurations, $J=\left(j_{1} \ldots j_{f}\right)$, where $f$ denotes the number of degrees of freedom and $Q \equiv\left(x_{1}, \ldots, x_{f}\right)^{T}$. The (unknown) singleparticle functions $\varphi_{j_{\kappa}}^{(\kappa)}$ are in turn represented in a fixed, primitive basis implemented on a grid. For indistinguishable particles as in our case, the single-particle functions for each degree $\kappa=1, \ldots, N$ are of course identical in both type and number $\left(\varphi_{j_{\kappa}}\right.$, with $\left.j_{\kappa} \leq n\right)$.

Note that in the above expansion, not only the coefficients $A_{J}$ are time-dependent, but so are the Hartree products $\Phi_{J}$. Using the Dirac-Frenkel variational principle, one can derive equations of motion for both $A_{J}, \Phi_{J}[28]$. Integrating this differential-equation system allows one to obtain the time evolution of the system via (3). Let us emphasize that the conceptual complication above offers an enormous advantage: the basis $\left\{\Phi_{J}(\cdot, t)\right\}$ is varia- 
tionally optimal at each time $t$. Thus it can be kept fairly small, rendering the procedure very efficient.

It goes without saying that the basis set above is not inherently permutation symmetric, as would be an obvious demand when dealing with bosons. However, the symmetry can be enforced by symmetrizing the coefficients $A_{J}$, even though this turns out to be unnecessary as far as the ground state is concerned, which is automatically bosonic [33].

The Heidelberg MCTDH package [34], which we use, incorporates a significant extension to the basic concept outlined so far, the so-called relaxation method [35]. MCTDH provides a way to not only propagate a wave packet, but also to obtain the lowest eigenstates of the system. The underlying idea is to propagate some wave function $\Psi_{0}$ by the non-unitary $e^{-H \tau}$ (propagation in imaginary time.) As $\tau \rightarrow \infty$, this automatically damps out any contribution but that stemming from the true ground state $|\mathbf{0}\rangle$,

$$
e^{-H \tau} \Psi_{0}=\sum_{J} e^{-E_{J} \tau}|J\rangle\left\langle J \mid \Psi_{0}\right\rangle
$$

In practice, one relies on a more sophisticated scheme termed improved relaxation. Here $\langle\Psi|H-E| \Psi\rangle$ is minimized with respect to both the coefficients $A_{J}$ and the configurations $\Phi_{J}$. The equations of motion thus obtained are then solved iteratively by first solving for $A_{J}(t)$ (by diagonalization of $\left(\left\langle\Phi_{J}|H| \Phi_{K}\right\rangle\right)$ with fixed $\left.\Phi_{J}\right)$ and then propagating $\Phi_{J}$ in imaginary time over a short period. The cycle will then be repeated.

As it stands, the effort of this method scales exponentially with the number of degrees of freedom, $n^{N}$. Just as an illustration, using 15 orbitals and $N=5$ requires $7.6 \cdot 10^{5}$ configurations $J$. This restricts our analysis in the current setup to about $N=O(10)$, depending on how decisive correlation effects are. If these are indeed essential, then it will turn out later that at least $n=N$ orbitals are needed for qualitative convergence alone, while the true behavior may necessitate about 15. By contrast, the dependence on the primitive basis, and thus on the grid points, is not as severe. In our case, the grid spacing should of course be small enough to sample the interaction potential, and we consider a basis set of 75 harmonic-oscillator functions. 


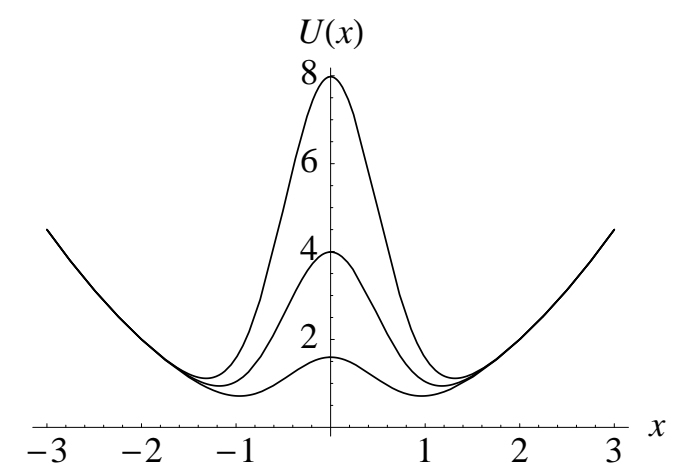

Figure 1: Sketch of the model potential $U(x)=\frac{1}{2} x^{2}+h \delta_{w}(x)$, consisting of a harmonic trap plus a normalized Gaussian of width $w=0.5$ and barrier strengths $h=2,5,10$.

\section{BOSONS IN A DOUBLE WELL}

In this as well as in the following section, we consider the ground-state properties of bosons in a double-well trap modeled by

$$
U(x)=\frac{1}{2} x^{2}+h \delta_{w}(x) .
$$

This potential is a superposition of a harmonic oscillator (HO), which it equals asymptotically, and a central barrier which splits the trap into two fragments (Fig. 11). The barrier is shaped as a normalized Gaussian $\delta_{w}$ of width $w$ and 'barrier strength' $h$.

As $w \rightarrow 0$, the effect of the barrier reduces to that of a mere boundary condition (since $\left.\delta_{w} \rightarrow \delta\right)$, and the corresponding one-particle problem can be solved analytically [12, 36]. Although this soluble borderline case presents a neat calibration, the exact width $w$ does not play a decisive role, as long as it is larger than the grid spacing and $w<1$ so as to confine the barrier's effect to the central region. We choose $w=0.5$ as a trade-off.

For $h=0$, the case of interacting bosons in a harmonic trap is reproduced. In Sec. IVA, we witness the transition from a simple, weakly interacting condensate $(g \rightarrow 0)$ to fragmentation and finally the Tonks-Girardeau limit $(g \rightarrow \infty)$. As $h \rightarrow \infty$, the energy barrier will greatly exceed the energy available to the atoms, and we end up with two isolated wells. Higher $g$ then affect only the fragmentation within each of these wells. In between, there is an interesting interplay between the 'static' barrier $(h)$ and 'dynamical barriers' in the form of inter-particle forces $(g)$. We study this intermediate regime on the examples of $h \in\{2,5\}$ in Sec. IVB 


\section{A. The reference case: $h=0$}

In the absence of a central barrier, we simply deal with a harmonic trap, which in the case of $N=2$ and point interactions $(\sigma \rightarrow 0)$ constitutes an exactly solvable problem [11]. Starting with all interactions turned off $(g=0)$, the ground-state solution is simply the

uncorrelated product of the HO-ground state, $\Psi=\phi_{0}^{\otimes N}$. As long as $g$ is small enough, this behavior remains qualitatively unaffected by interactions, which only alter the shape of the single-particle functions via a mean field (the Gross-Pitaevskii regime). Increasing $g$ has the effect of continually depleting the interaction regions $\left\{x_{i}=x_{j}\right\}$; the repulsion forces the atoms to isolate each other, a process referred to as fragmentation (cf. Sec. IVD for a rigorous account of this). Driven to extremes as $g \rightarrow \infty$, this fragmentation saturates in a socalled fermionized state. In that case the Bose-Fermi mapping [6] asserts that letting $g \rightarrow \infty$ emulates the effects of the Pauli exclusion principle, and the bosons have accomplished to minimize their density overlap.

These qualitative considerations materialize in the reduced densities of the ground-state. In the 2-particle density $\rho_{2}\left(x_{1}, x_{2}\right)$ - the diagonal kernel of the reduced density operator

$$
\rho_{2}:=\operatorname{tr}_{3 . . N}|\Psi\rangle\langle\Psi|
$$

which yields the probability density of simultaneously finding any two particles at $x_{1}$ and $x_{2}$ - the correlation diagonal $\left\{x_{1}=x_{2}\right\}$ forms an ever deeper dip (the 'correlation hole'). This is illustrated in Fig. 2 for $g=0.2$ and $g=194$. These represent the borderline cases of a weakly interacting system and the strong-correlation regime, respectively. In the former case, the density is simply Gaussian-like. For large enough repulsion, in turn, the density resembles more and more the checkerboard pattern produced by a (polarized) fermionic state.

The 1-particle density $\rho_{1}(x)$ offers another tool to visualize the fermionization process. Fig. 3 gives an impression of how the profile changes from a harmonic one $(g \ll 1)$, to one flattened due to repulsion for mediate $g$, and finally to the Tonks-Girardeau limit $(g \geq 15)$, where $N$ humps emerge, mimicking the fermionic behavior. 


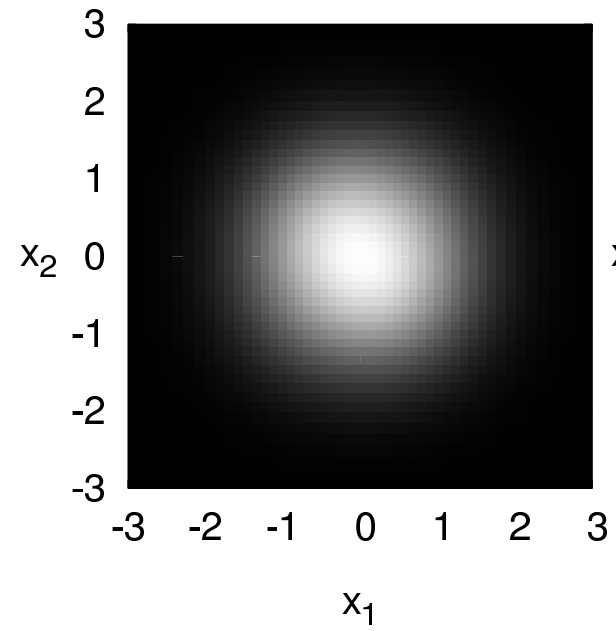

(a)

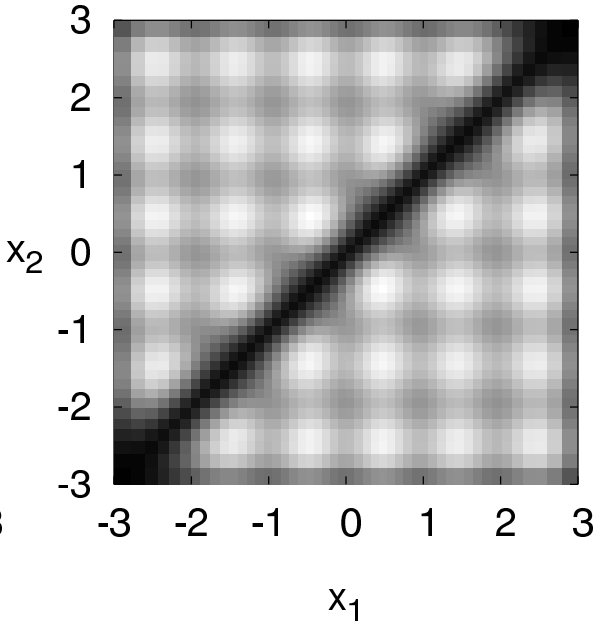

(b)

Figure 2: 2-particle density $\rho_{2}\left(x_{1}, x_{2}\right)$ for a harmonic trap $(N=6)$ for (a) $g=0.2$ and (b) $g=194$.

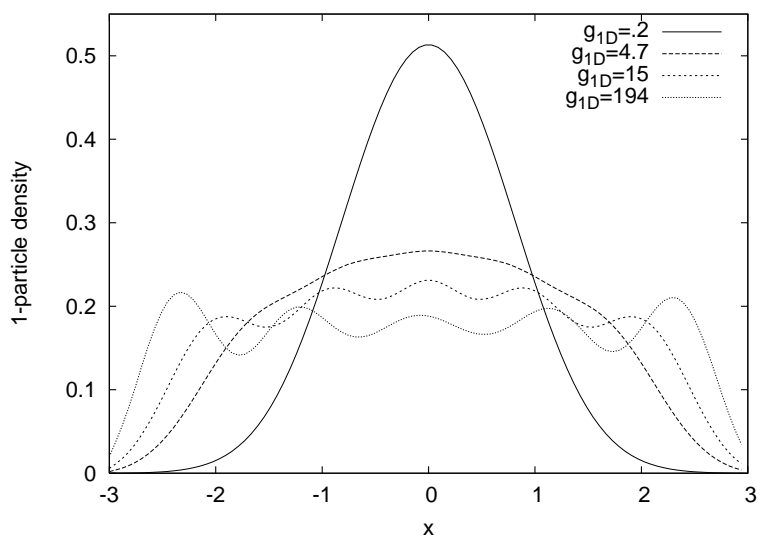

Figure 3: 1-particle density $\rho_{1}(x)$ for a harmonic trap $(N=5)$ for different interactions $g_{1 \mathrm{D}}$. Note how the profile changes from a weakly interacting one $(g=0.2)$ to a flattened one due to fragmentation, and finally to a fermionized profile featuring $N$ humps $(g \geq 15)$.

\section{B. Central barrier $h>0$}

We now introduce a central barrier $h \delta_{w}(x)$, so as to turn the harmonic trap into a double well. Upon increasing $g$, there are now two competing effects, exemplified on the 2-particle density (Fig. 4): For small enough $g$, the barrier $h$ dispels the atoms from the center $x=0$. This state is uncorrelated: they are localized in both wells regardless of whether there 


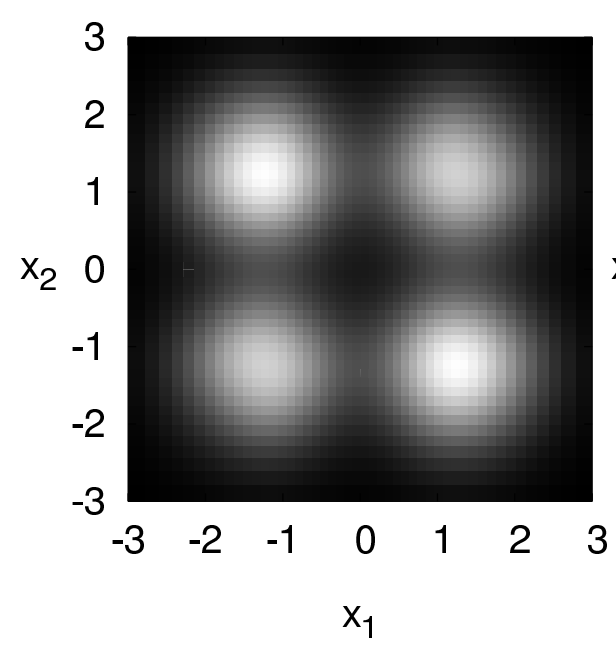

(a)

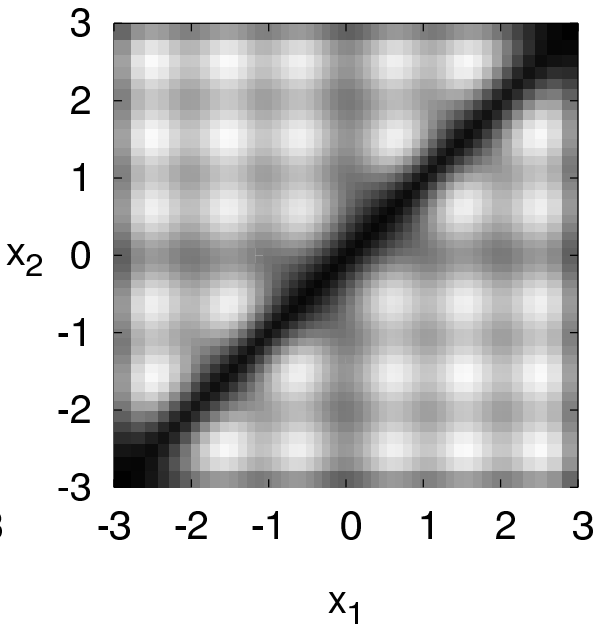

(b)

Figure 4: 2-particle density $\rho_{2}\left(x_{1}, x_{2}\right)$ for a double-well trap with barrier strength $h=5(N=6)$. (a) The weakly interacting limit $g=0.2$ (b) The complementary case of fermionization, $g=194$.

are already any other ones. Indeed, in Fig. 4(a) the diagonal $\left\{x_{1}=x_{2}\right\}$ shows only a tiny depletion due to repulsion. In the complementary case $g \rightarrow \infty$, the barrier will be almost outweighed by the inter-particle repulsion. In this Tonks-Girardeau limit, the atoms distribute so as to minimize their density overlap, at the price of an additional potential energy in the barrier region. Hence the two-particle density in Fig. 4(b) differs from the harmonic counterpart only in the stripes along $x_{1 / 2}=0$, which indicate suppression in the central-barrier region. The question as to what happens in the intermediate region requires a distinction between even and odd particle numbers; it will be the focus of the ensuing paragraphs.

\section{Even $N$ : assisted fragmentation}

For an even number of atoms - in what follows $N \in\{2,4,6\}$ - they initially (at $g=0$ ) populate each well with $N / 2$ atoms. Upon raising the interaction strength, they seek to isolate each other, which can best be done by having a fragmentation within each well, interfering only little with the central barrier. This situation is depicted in Fig. 5 for four 


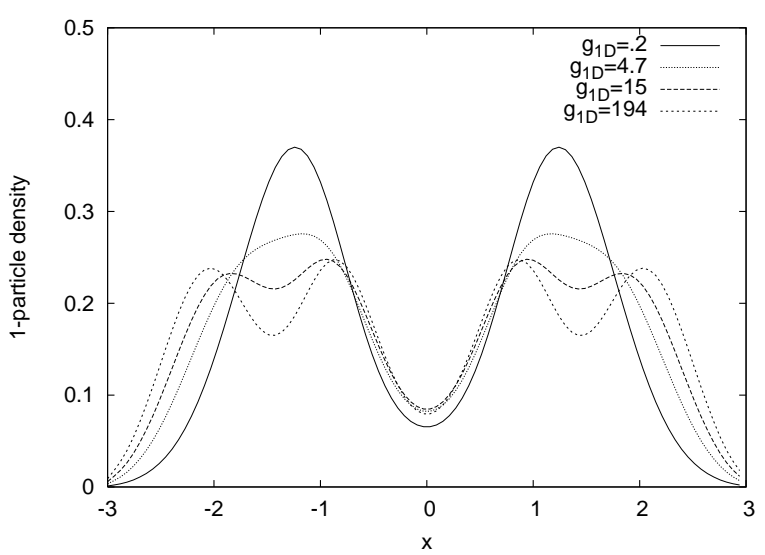

(a)

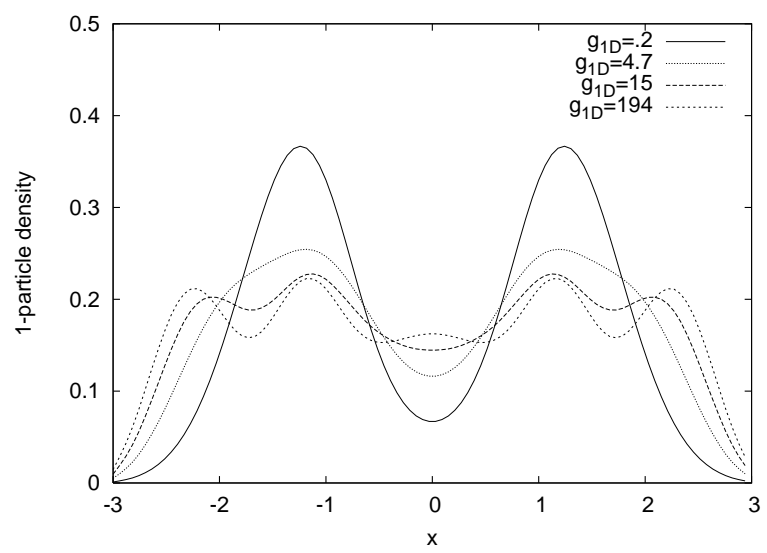

(b)

Figure 5: 1-particle density in a double well with $h=5$ : Even vs. odd number of atoms. (a) For $N=4$ atoms, the fragmentation process is in part supported by the central barrier. By contrast, (b) indicates in what sense fermionization is suppressed for an odd number $(N=5)$, since by symmetry one particle should eventually reside in the center.

atoms. The plots for $g=4.7,15$ reflect the tipping from a coherent state to fragmentation. For high enough $g$, the accessible energy approaches that of the barrier, $N h / \sqrt{2 \pi} w$, so that tunneling becomes more and more dominant. Beyond that point, the density profile is expected to resemble that of a purely harmonic trap with slightly suppressed amplitude in the barrier region. In particular, for any finite $h$, the Tonks-Girardeau limit will be of a generic form in that it exhibits $N$ density maxima. In conclusion, one might say that the fragmentation here is assisted insofar as the central barrier helps isolate the ensemble, a statement put more precisely in IVD.

\section{Odd $N$ : delayed fragmentation}

In the case of an odd number $(N=3,5)$, the situation differs. At $g=0$, the atoms are again coherently distributed over the two wells. As we strengthen the interactions, they try to enlarge their distance-but by symmetry, this can now only be done by placing one particle at $x=0$, which in turn is impeded by the barrier (cf. Fig. 5b). In other words, the system will have to pay the added interaction energy and distribute the extra particle over the two wells, until the former one becomes high enough to afford the place in the 
central-barrier zone. In that sense, the fragmentation is attenuated by the double-well trap.

\section{Ground-state energy}

One central aspect of our analyses is the ground-state energy. Fig. 6 depicts a typical evolution, $E(g)$, as a function of the coupling strength. As suggested above, the $g \rightarrow \infty$ state is isomorphic to a non-interacting fermionic state. In this light, also the ground-state energy $E(g)$ (Fig. 6) may be interpreted as connecting the free bosonic $(g=0)$ and the free fermionic value, corresponding to the saturation as $g \rightarrow \infty$.

The effect of the interaction at $g=0$ can be measured by the slope

$$
\frac{d E}{d g}(0)=\frac{N(N-1)}{2}\left\langle 00\left|\delta_{\sigma}\left(x_{1}-x_{2}\right)\right| 00\right\rangle \stackrel{\sigma \rightarrow 0}{\sim} \frac{N(N-1)}{2} \int\left|\phi_{0}(x)\right|^{4} d x
$$

given by the density overlap of two atoms in the non-interacting ground state. The centered harmonic-oscillator orbital $\phi_{\mathrm{HO}}$ by construction has a low curvature (i.e., kinetic energy), thus producing a rather high density overlap. It is thus more susceptible to the onset of interactions. By contrast, the presence of a central potential-energy barrier $(h \rightarrow \infty)$ evokes an orbital $\phi_{\text {DW }}$ delocalized in both wells. Its density overlap in turn will be smaller, which can be seen schematically by assuming for a moment that $\phi_{\mathrm{DW}}(x) \sim \frac{1}{\sqrt{2}}\left[\phi_{\mathrm{HO}}\left(x-x_{0}\right)+\phi_{\mathrm{HO}}\left(x+x_{0}\right)\right]$ is built from a HO orbital centered in both minima $\pm x_{0}$. Neglecting the density overlap between the right- and left-hand contributions, $\int\left|\phi_{\mathrm{DW}}\right|^{4} \simeq \frac{1}{2} \int\left|\phi_{\mathrm{HO}}\right|^{4}$, suggesting that in a double well, the atoms will feel a lesser effect when interactions are turned on. This can be seen in Fig. 6]

\section{The role of fragmentation}

We have so far relied on a more intuitive notion of fragmentation. It is natural to ask whether some of our previous assertions can be put more quantitatively. This we seek to do in the present subsection, not only to highlight some deeper concepts, but also to show why a numerically exact approach is so vital.

It has been argued that ever stronger interactions introduce correlations to the system, viz., the one-particle Hamiltonian will be no longer dominant, but the influence of the two-particle operator $V$ takes over. The latter one imprints explicit two-body correlation 


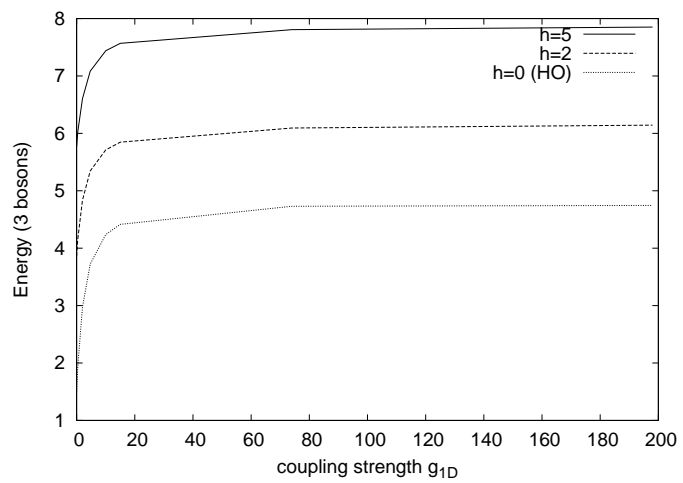

Figure 6: Energy $E(g)$ for the case $N=3$. Note the slightly different effect of the interaction, measured by the slope at $g=0$, for different barrier strengths $h=0$ (harmonic trap) and $h=2,5$. The saturation as $g \rightarrow \infty$ corresponds about to a fermionized state.

terms on the eigenvector, as illustrated, for instance, on the example of two atoms in a trap [11, 12] or the fermionization limit $g \rightarrow \infty$ solvable for any $N$ [37] starting from the Bose-Fermi mapping,

$$
\Psi(Q) \propto e^{-|Q|^{2} / 2} \prod_{1 \leq i<j \leq N}\left|x_{i}-x_{j}\right| .
$$

Technically speaking, it is therefore far from clear how long $\Psi$ can be well approximated by states composed of one-particle functions, let alone a single such configuration as in mean-field approaches (see [10] and references therein).

Being exact, our method offers a handle on these convergence questions, based on a criterion put forward by Penrose [33]. Consider the spectral decomposition of the oneparticle density matrix

$$
\rho_{1}=\operatorname{tr}_{2 . . N}|\Psi\rangle\left\langle\Psi\left|\equiv \sum_{a} n_{a}\right| \phi_{a}\right\rangle\left\langle\phi_{a}\right|,
$$

where $n_{a} \in[0,1]$ is said to be the population of the natural orbital $\phi_{a}$. Obviously, if all $n_{a}^{\prime} \equiv n_{a} N \in \mathbb{N}\left(\sum_{a} n_{a}^{\prime}=N\right)$, then the density may be mapped to the (non-interacting) number state $\left|n_{0}^{\prime}, n_{1}^{\prime}, \ldots\right\rangle$ based on the natural one-particle basis; for non-integer values it extends that concept. In particular, the highest such occupation, $n_{0}$, may serve as a measure of non-fragmentation: for $n_{0}=1$, a simple condensate is recovered. This is the well-known borderline case of the Gross-Pitaevskii eq.: as $g \rightarrow 0, \rho_{1} \rightarrow\left|\phi_{0}\right\rangle\left\langle\phi_{0}\right|[38]$. The complementary fermionization limit $(g \rightarrow \infty)$ has been investigated semi-analytically drawing on the Fermi/Bose mapping, yielding $n_{0} \simeq N^{-.41}$ for a harmonic trap [37]. 


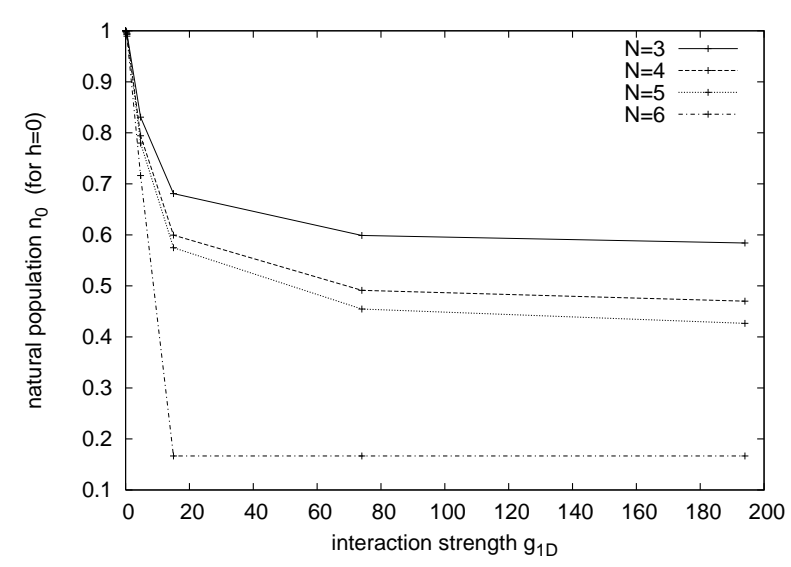

(a)

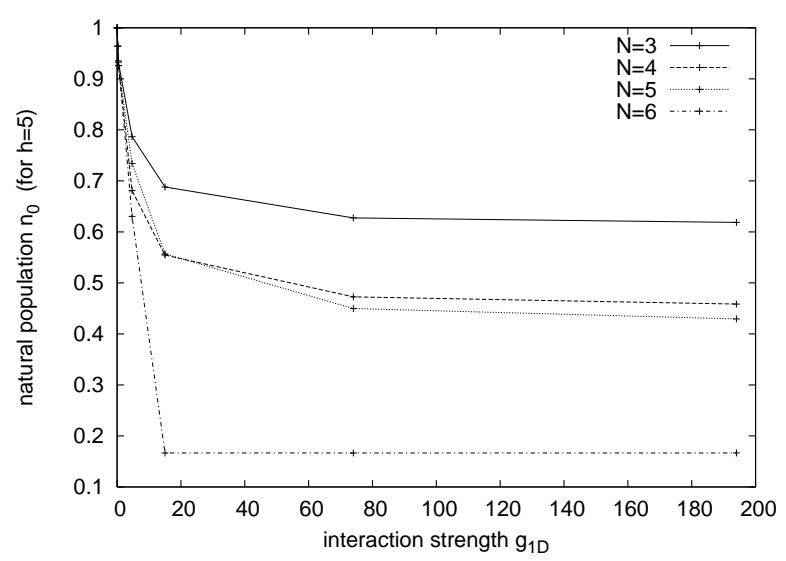

(b)

Figure 7: Maximum natural population $n_{0}(g)$ for (a) a harmonic trap $(h=0)$ and (b) a double well $(h=5)$. For even $N$, the fragmentation is enhanced by the higher barrier, whereas for odd $N$ it is slowed.

In between those extremes, a thorough many-body treatment is indispensable. This is illustrated in Fig. 7 , where $n_{0}(g)$ is plotted. Observe that, for one thing, the high-correlation value $n_{0}(g \rightarrow \infty)$ is much too low for $N=6$ compared with the above value $n_{0} \simeq .48$, a numerical effect explained below. Moreover, the lines for $N=4$ and $N=5$ in the doublewell trap (Fig. 7b) are partly reversed as compared to the harmonic trap, while the $N=3$ value of $n_{0}$ is shifted upward. This reflects the suppression of fragmentation for odd atom numbers with respect to even $N$.

The role played by correlations may also be highlighted by the number of single-particle functions needed in a calculation, $n$ (cf. III). It also determines how many terms in the spectral expansion of $\rho_{1}$ (Eq. 4) are included. Figures 8 (a-c) show the convergence of the ground-state energy of $N=6$ bosons as $n$ is varied. For rather low $g=.406$, the convergence for the harmonic trap is fairly smooth, and the variations are altogether relatively small. This asserts that Gross-Pitaevskii works qualitatively well, though it may already take many orbitals to achieve a high accuracy. For a double well with $h=5$, the convergence is much more abrupt. Adding just another orbital, $n=2$, lowers the energy drastically in comparison with any further refinements. This is intelligible, regarding the fact that two-mode models based essentially on the anti-/symmetric orbitals of a double well are widely used in that 


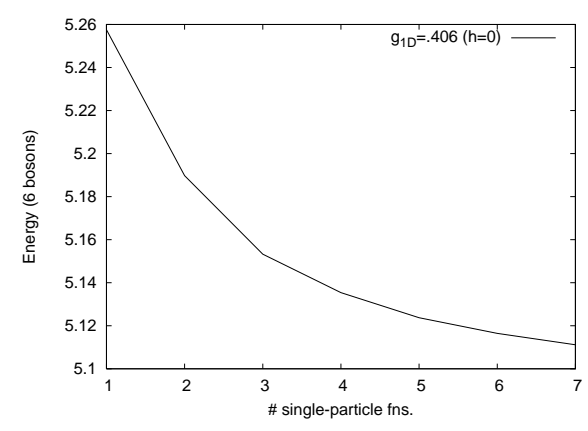

(a)

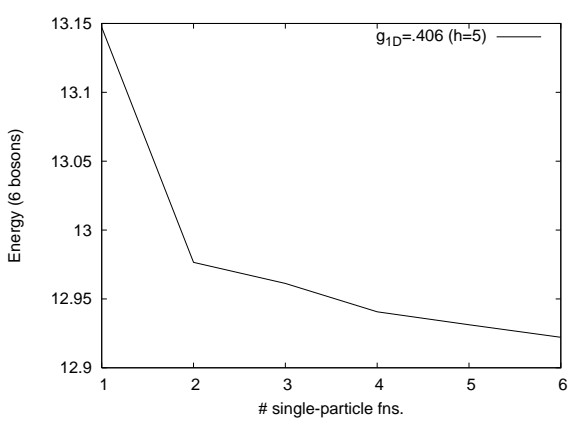

(b)

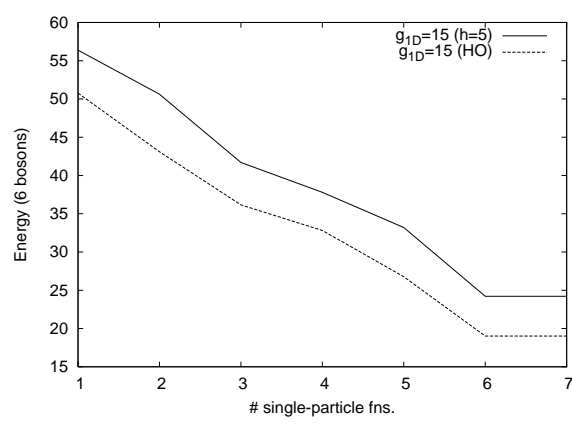

(c)

Figure 8: Convergence of the energy $E(n)$ in the number of single-particle functions $n$ for six bosons. For weak interaction $g=.406$, the difference between the harmonic (a) and the split trap (b) is conspicuous, though in both cases Gross-Pitaevskii $(n=1)$ is not too far off. As opposed to that, the onset of fermionization $(g=15)$ wipes out that difference up to a residual energy offset due to the central barrier (c). Here $n=N$ orbitals are needed to be at least qualitatively correct.

context.

Fig. 8(c) in turn casts a light on the strong-correlation regime, $g=15$ (in fact its ground state for $h=5$ is already atop the central energy barrier.) Here the lines $E(n)$ for $h=0,5$ are almost parallel, shifted only by the energy offset of about $h \sim \int h \delta_{w}(x) d x$. Clearly simple mean-field theory is off by more than a factor of 2, as it drastically overestimates the interaction energy and fails to reproduce the characteristic Tonks-Girardeau profile with $N$ humps. Just when $n=N=6$, the convergence suddenly settles, and the qualitatively correct behavior can be observed. Interestingly enough, adding another orbital, $n=7$, has virtually no effect on that energy scale: the first $N$ natural occupations all turn out to be $n_{0}=1 / N$, corresponding to a number state $\left|1_{0}, \ldots, 1_{N-1}\right\rangle$ in the natural-orbital basis. This is of course quantitatively incorrect in light of the expected behavior $n_{0} \sim N^{-0.41}$, which really can be recovered if $n \gtrsim 15$ single-particle functions are included. But apparently that ground state seems to be a somewhat stable intermediary solution in the fermionization limit if the subspace $\operatorname{span}\left\{\Phi_{J} \mid j_{\kappa} \leq n\right\}$ is taken too narrow. This provides an insightful link to multi-orbital approaches [9, 10].

Let us remark that the criterion employed above is conventional and amenable, but not imperative. Indeed, the two-particle density $\rho_{2}$ by nature reveals correlation effects even 
more clearly.

\section{INHOMOGENEOUS INTERACTIONS}

We have so far relied on the assumption of homogeneous two-particle forces. These are invariant under global translations and thus depend on $x_{i}-x_{j}$ alone. While this premise is most natural from a fundamental point of view, we should keep in mind that our description is not a fully microscopic one, even if we ignore the internal structure of the underlying atoms. Rather, it is an effective model stripped not only of the transverse degrees of freedom, but of course also of the electromagnetic fields that manipulate both external and inter-particle forces.

With this in mind, it appears legitimate to conceive situations where the strength of the interactions depends in addition on the position where the collision takes place, as was done in a mean-field framework in Ref. [39] (see also citations therein). This may be induced by means of a Feshbach resonance, tuning $a_{0}(\mathbf{B})$ by adding a spatial dependence to the magnetic field. In our one-dimensional setting, it seems even more convenient to exploit the parametric dependence on the transverse subsystem, and modify $a_{\perp}$ locally so as to imprint a spatial dependence on $g_{1 \mathrm{D}}$.

Without reference to the specific realization and its concrete experimental constraints, we perform a case study where a generic model for the inhomogeneity is assumed to begin with. This model will be presented in Sec. VA The interplay of that dynamical inhomogeneity with the external forces will be studied for a harmonic ( $\mathrm{VB}$ ) and a double-well trap ( $\mathrm{VC}$ ).

\section{A. Model interaction}

Whereas modeling a position-dependent interaction in a mean-field description (as in [39]) is straightforward, since one only has an effective one-particle problem, one faces a conceptual problem when using a many-body framework. In general, the coupling would depend on both participants $x_{i}, x_{j}$, which is technically possible if somewhat awkward. For

it to make sense intuitively, we require that its modulation lengthscale be much larger than the 'radius' of collision, $\sigma$.

With this is mind, it is natural to model our interaction in terms of the respective relative 
coordinate $r:=x_{i}-x_{j}$ (for fixed $i, j$ ) and-in order to keep $V$ formally symmetric - the center of mass $2 R:=x_{i}+x_{j}$ :

$$
V(r, R)=g(R) \delta_{\sigma}(r)
$$

The demand that $g$ change slowly over the support of $V$ can be cast as

$$
\left\|\frac{g}{g^{\prime}}\right\|_{\infty} \gg \sigma
$$

in the supremum norm.

There are various possibilities just what scenario should be examined, be it some kind of collision-enhanced tunneling or dynamical self-trapping [39]. We concentrate on a specific model where $g$ is essentially imbalanced between the right- and left-hand sides of the trap (Fig. 9):

$$
g(R)=g_{0}\left[1+\alpha \tanh \left(\frac{R}{L}\right)\right] .
$$

This signifies that for $|R| \gg L$, the coupling takes on the asymptotic values

$$
g_{ \pm} \equiv \lim _{|R| \rightarrow \pm \infty} g(R)=g_{0}(1 \pm \alpha)
$$

while it changes on a scale of $L$ near the trap's center about $g_{0}$. The parameter $\alpha$ regulates both the relative difference between the asymptotic strengths and their ratio:

$$
\begin{aligned}
\Delta g & \equiv\left|g_{ \pm}-g_{0}\right|=g_{0} \alpha, \\
\frac{g_{+}}{g_{-}} & =\frac{1+\alpha}{1-\alpha} .
\end{aligned}
$$

Eq. (5) can be met if $L \gg \sigma \alpha$, which is effortlessly fulfilled if we choose $L=1$ for convenience.

\section{B. The reference case: $h=0$}

Generally speaking, the ground state of atoms immersed in a harmonic trap will be centered at the trap's bottom, assuming that we start with a weakly interacting ensemble. Hence the modulation of the coupling strength $g$ beyond the center will pass them largely unnoticed. It is only for strong enough repulsive interaction-where fragmentation sets inthat the density profile will start to split and shift partly outward, thus experiencing an asymmetry.

This picture is supported by our calculations, as demonstrated in Figure 10 for $N=5$ atoms. For low enough $g_{0}=.4$, measuring the average interaction strength, the harmonic 


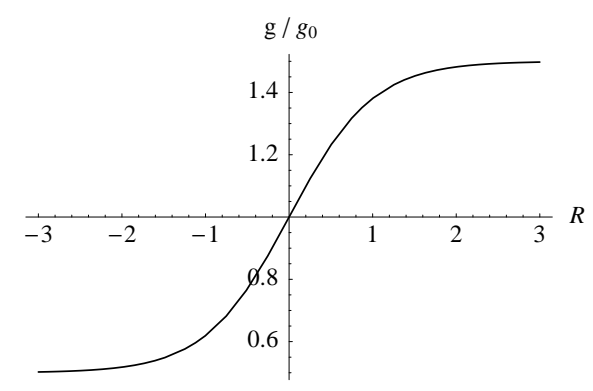

Figure 9: Our model of the position-dependent coupling $g(R) / g_{0}=1+\alpha \tanh \left(\frac{R}{L}\right)$. The relative modulation, here $\alpha=0.5$, determines the asymptotic difference from the average value $g_{0}$, while the modulation length $L=1$ shall remain fixed.

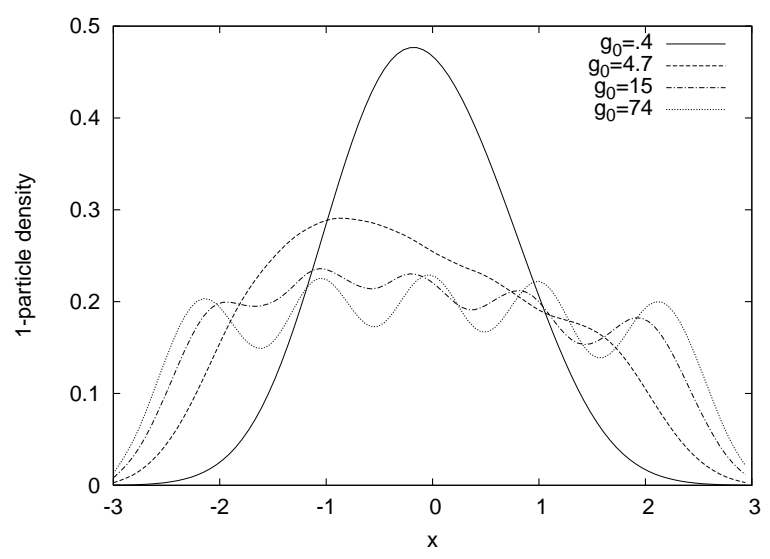

Figure 10: 1-particle densities $\rho_{1}(x)$ for a harmonic trap $(N=5)$ in the case of inhomogeneous interactions, here $\alpha=.5$. The profile features an imbalance for smaller interactions $g_{0}$, where the wave packet is centered too much to sample the modulation of $g(R)$. When fragmentation sets in, the profile splits and the asymmetry becomes more distinct. In the fermionization limit, the energy costs of an imbalance become too large to keep it up.

profile is barely altered from the homogeneous case $\alpha=0$. An imbalance is noticed for medium $g_{0}=4.7$ : the atoms are now able to sample the modulation of the coupling strength and find it more inexpensive to locate in the less repulsive zone $x<0\left(g_{-}\right)$. However, this effect ceases as the repulsion becomes larger $(g \geq 15)$. This may be interpreted as follows: the energetical costs for concentrating several particles near one spot are soaring, and this in total eventually outweighs the relative energy savings reached by an imbalance.

A look into the two-body correlations $\rho_{2}\left(x_{1}, x_{2}\right)$ in Fig. [1] helps us clarify what happens. 


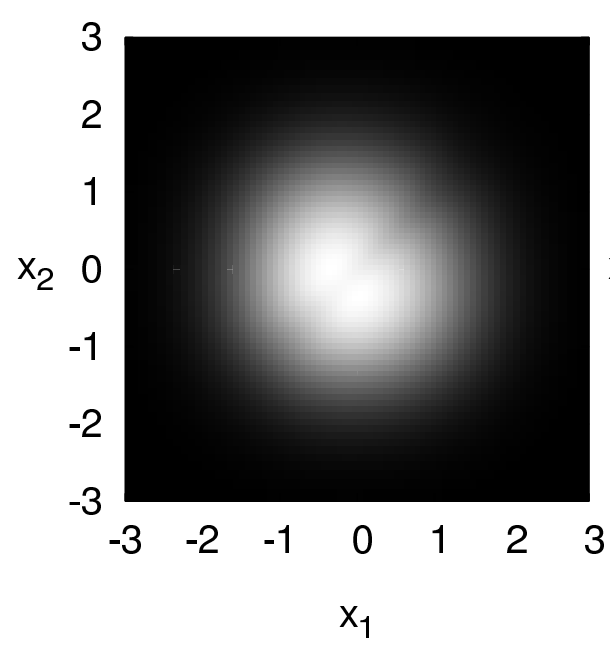

(a)

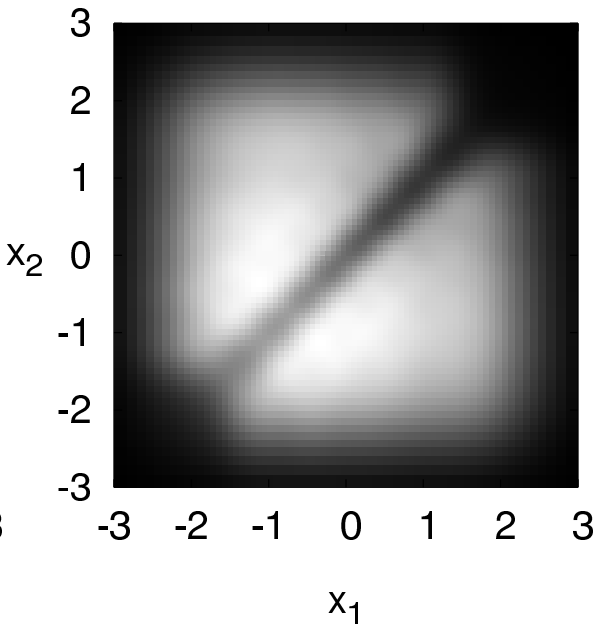

(b)

Figure 11: 2-particle density for a harmonic trap in the presence of inhomogeneous interactions $(N=5)$. (a) For $g=0.4$, the packet is localized about the center, thus widely ignoring the modulation. (b) When fragmentation sets in $(g=15)$, it starts to delocalize and consequently shifts to $R<0$.

Along the correlation diagonal $\left\{x_{1}=x_{2}\right\}, R=x_{1 / 2}$ holds. It is here that the modulation can have an impact, whereas for $x_{1}=-x_{2}, g(R)=g(0)=g_{0}$ as usual. Clearly the density on the diagonal $\left\{x_{1}=x_{2}\right\}$ must be spread enough for the modulation to become effective. This is not the case for small interactions. Indeed, for $g=0.4$, the packet is localized about the center, thus widely ignoring the modulation. Yet for medium $g_{0}$ (Fig. 11b), the repulsion-driven broadening has become distinct enough so that the ground states exhibits some left-right asymmetry. For strong fragmentation, the correlation diagonal will in turn be fully depleted, so obviously the atoms can no longer realize the modulation, and the very premise of an inhomogeneity-based displacement has become obsolete.

The above findings are nicely wrapped up in Fig. 12, showing graphs of $\langle x\rangle=\operatorname{tr}\left(\rho_{1} x\right)$ as a function of $g_{0}$ for $N=5$. For $\alpha=0$, and of course for $g_{0}=0$, no modulation exists and, by symmetry, $\langle x\rangle=0$. Notably, the same goes for $g_{0} \rightarrow \infty$, when the correlation diagonal is depleted as delineated above, even though the displacement will vanish only very slowly. There is a trade-off in between for which $\langle x\rangle$ becomes extremal. The value where this occurs, $g_{0}^{\star}(\alpha)$, depends only weakly on the relative modulation $\alpha$-despite the fact that the maximum ground-state displacement $-\langle x\rangle^{\star}$ will of course increase monotonically with 


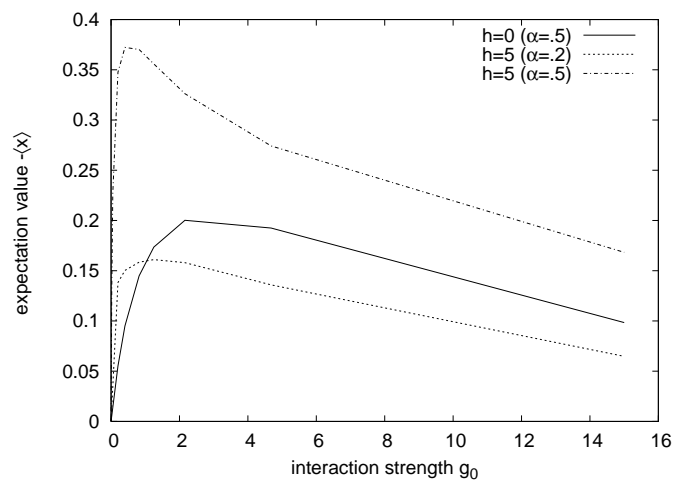

Figure 12: The ground-state displacement $-\langle x\rangle$ as a function of the average interaction $g_{0}(N=5)$. Its universal behavior is an increase up to a maximum value followed by a slow decay. The increase at $g_{0}=0$ is strongly enhanced in the presence of a barrier $h>0$, while for the purely harmonic trap $(h=0)$, it is rather slow. Of course the maximum itself is much more pronounced for higher modulations $\alpha$, while being absent in the homogeneous case $\alpha=0$.

$\alpha$.

\section{Central barrier $h>0$}

In the presence of a sufficiently strong barrier, the situation is a different one. To begin with $\left(g_{0}=0\right)$, the atoms are not centered as before but rather coherently distributed over the two wells. Hence, upon switching on the inhomogeneous interaction, they can immediately feel the full impact of its modulation on both sides. For finite barrier strength $h$, they can then re-distribute so as to find a compromise between minimum repulsion and potential energy. Even though this mechanism is universal for any particle number, we will lay it out for both even and odd $N$ so as to keep a link to the reference case $\alpha=0$.

\section{Even $N$}

The above process is illustrated in Fig. 13, which evidences an immediate shift from the right well to the left one, where the repulsion is weaker. This still corresponds to the Gross-Pitaevskii regime of a single dominant orbital: there is no correlation hole; in fact the probability density of finding both particles in the left well, $\rho_{2}\left(-x_{0},-x_{0}\right)$, may even be 


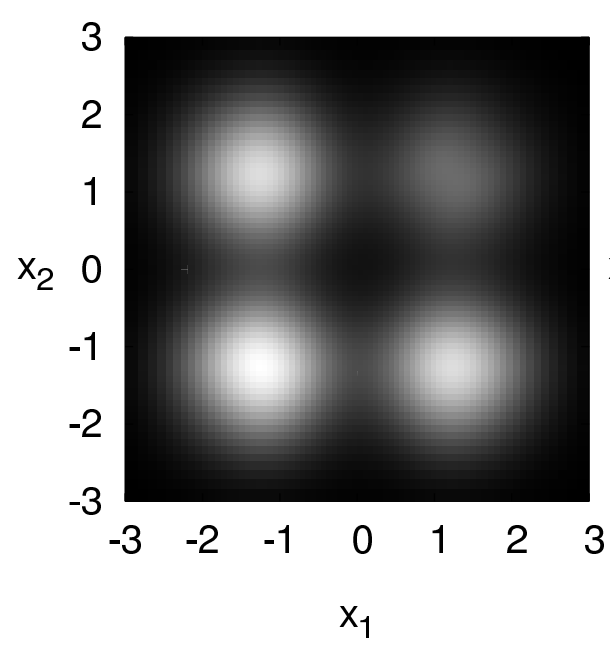

(a)

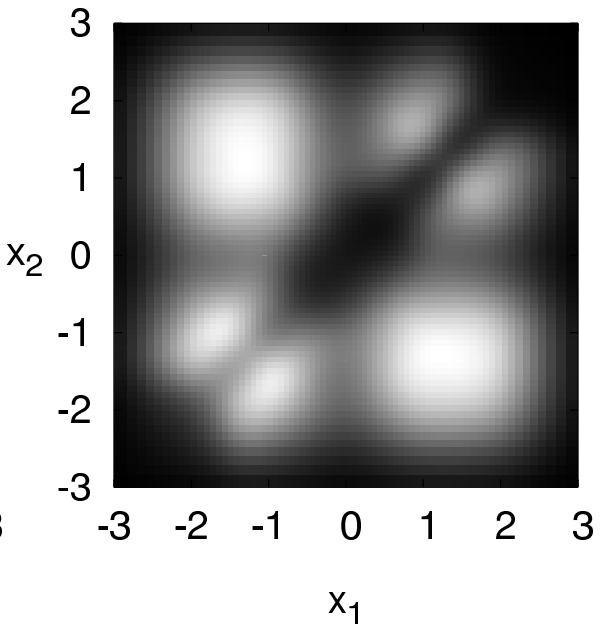

(b)

Figure 13: 2-particle density for $N=4$ bosons in a double-well trap $(h=5)$ and with inhomogeneous interactions $(\alpha=.5)$. (a) Already for $g=0.2$, the probability of finding any two atoms in the left well is significantly enhanced. (b) At the onset of fragmentation $(g=15)$, the diagonal $\left\{x_{1}=x_{2}\right\}$ is being depleted.

larger than that for separation, $\rho_{2}\left( \pm x_{0}, \mp x_{0}\right)$. As the interaction passes a critical strength, fragmentation sets in, somewhat more pronounced on the right-hand side (Fig. 13b). Note how the diagonal $\left\{x_{1}=x_{2}\right\}$ is being emptied, signifying the incipient destruction of the imbalance.

This reflects in the 1-particle density displayed in Fig. 14. The density is almost 'instantaneously' shuffled from the right to the left. In the curve for $g_{0}=4.7$, it becomes apparent that the fragmentation essentially kicks in separately for both wells, where only the right well exhibits the typical repulsion-induced split-up. As asserted already for the harmonic reference case $(h=0)$, the modulation becomes marginal relative to the overall fermionization process. This may also be discerned here: for an even number of bosons, the asymmetric effect fades, and as in Sec. IVB, the fragmentation is assisted by the central barrier insofar as it now enters separately in the two wells. 


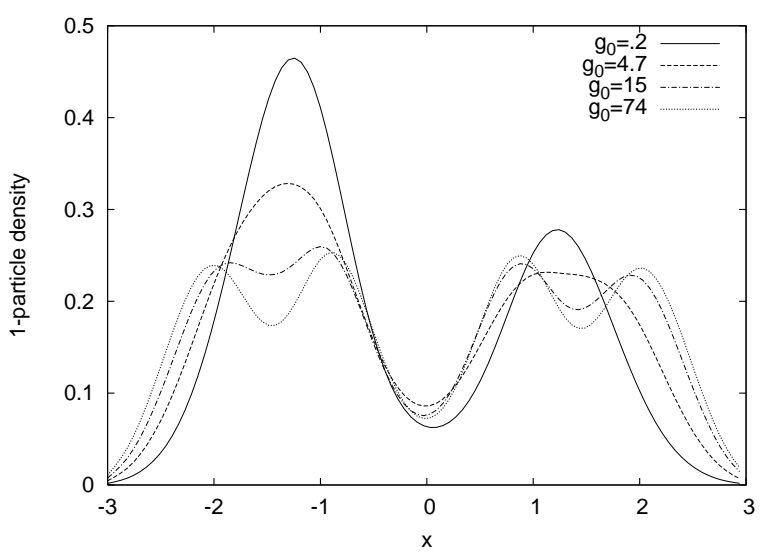

(a) Four atoms

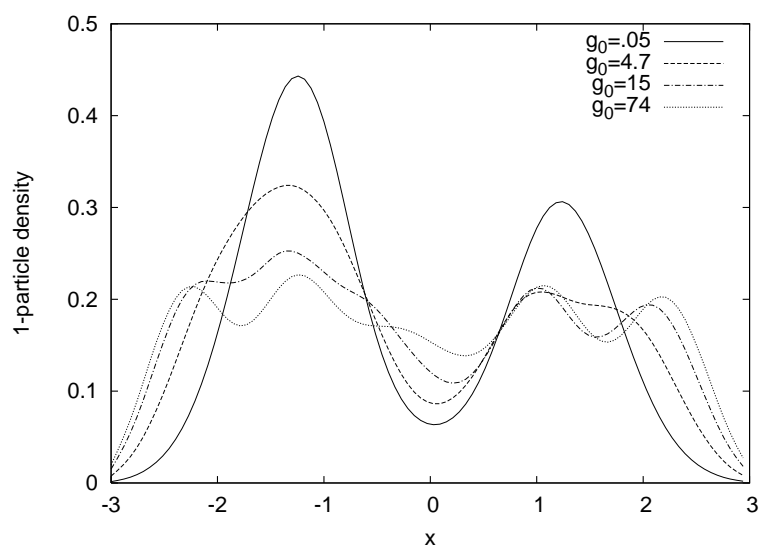

(b) Five atoms

Figure 14: 1-particle density for a double well $(h=5)$ and modulated coupling strength $(\alpha=.5)$ : Even vs. odd number of atoms. In both cases, fragmentation essentially sets in separately in each well (e.g., see $g_{0}=4.7$ ). However, for $N=4$ (a), the fermionization process is again supported by the central barrier, while (b) suggests that for $N=5$ the extra particle can now be temporarily accommodated by the left well.

\section{Odd $N$}

The behavior here is wholly analogous to that evidenced in Fig. 13. On the correlation diagonal, the density again experiences an asymmetry even for tiny $g>0$, where the meanfield behavior is still virulent. For a strong enough modulation, say $\alpha=.5$, coincidence of two atoms in the left well $\left(x_{1 / 2}=-x_{0}\right)$ is even enhanced with respect to separation $\left(x_{1}=-x_{2}\right)$, while coincidence in the right well is extinguished very quickly. At a certain point, fragmentation sets in, which eventually evolves into fermionization.

It should be noted that the characteristic influence of the even atom number is on the transition to fermionization. Recall that this was hampered for homogeneous interactions owing to symmetry, which imposed that one particle had to reside near the center, $x=0$. This no longer holds here, and in fact, Fig.14(b) unveils that the spare particle is practically accommodated in the left well.

The nature of the ground-state displacement is again summarized in the graph of $-\langle x\rangle$ (Fig. 12). While the harmonic system turned out to be rather irresponsive to $g_{0}$, the displacement now exhibits a dramatic increase with raising $g_{0}$, as laid out above. It finds a 
maximum, which corresponds to the trade-off between localizing in the left well and maximum spreading. As before, the modulation $\alpha$ does not so much alter the critical $g_{0}^{\star}(\alpha)$, but of course makes for a stronger maximum displacement $\langle-x\rangle^{\star}$. The displacement decreases again slowly beyond that point. A notable side effect is that the displacement in the presence of a central barrier may in fact drop below the one without it, although of course this can only happen if the modulation $\alpha$ was smaller to begin with. That is simply because the double well, favoring the delocalization of the atoms, not only supports the modulation's effect, but also accelerates fragmentation and hence - eventually - destruction of the asymmetry.

\section{CONCLUSION AND OUTLOOK}

We have studied the numerically exact ground state of $N$ one-dimensional bosons in double-well traps, where the atoms interact repulsively via a short-ranged potential whose strength as well as its spatial modulation could be tuned. Our approach relied on the MultiConfiguration Time-Dependent Hartree method, a wave-packet dynamics tool known for its efficiency in higher dimensions. This allowed us to study the interplay between different heights of the barrier separating both wells, on the one hand, and different interaction strengths as well as spatial modulations in all relevant regimes in a numerically exact fashion.

For standard, homogeneous interactions, we have witnessed the transition from the weakcoupling mean-field regime to fragmentation and finally to a fermionized ground state for very large repulsion. Absent any barrier, this process requires sufficient interaction energies so as to compensate the one-particle (kinetic and potential) energy added by a fragmentation; also see [10]. Its signature is a broadening and eventually the appearance of $N$ humps in the density profile, plus a 'correlation hole' in the two-particle density. As demonstrated, this also reflects in the relative occupation of the dominant natural orbital, $n_{0}$, which reduces from unity to order of $1 / \sqrt{N}$ as the interaction is increased. As we turn the harmonic trap into a double well, then well below the barrier energy the fragmentation essentially takes place separately in each well, whereas way above the barrier, the situation resembles that in the harmonic trap. In particular, we find that for even $N$, fermionization is assisted, while it is impeded for odd $N$, when by symmetry one particle should be distributed over the barrier region.

We have also tackled the question of inhomogeneous effective interactions, insomuch 
as the coupling strength is assumed to be larger on one side of the trap. We have found the ground state to be displaced toward the side where repulsive forces are weaker. This displacement can be enhanced by stronger modulations, whereas the optimal interaction strength needed to achieve it can be lowered primarily by higher barriers. It falls off for stronger interactions as the fermionization destructs any imbalance effects.

This work can be seen as a model study of the effects of inhomogeneous interactions, in particular a setup with an imbalance inducing a parity violation. It can in principle be extended to higher particle numbers, although this was not done here with an eye toward time and computational effort. On the other hand, there are also plenty of promising other configurations that come into question, such as enhancing the transmission through a barrier by modulating the interaction strength accordingly or using more than two wells so as to separate single atoms from the system. Of course, to address these problems, eventually a time-dependent simulation is mandatory to gain insight into realistic situations, while the extension to more dimensions may become inevitable.

\section{Acknowledgments}

Financial support from the Landesstiftung Baden-Württemberg in the framework of the project 'Mesoscopics and atom optics of small ensembles of ultracold atoms' is gratefully acknowledged by PS and SZ. These two authors also appreciate A. Streltsov's helpful comments and thank O. Alon for illuminating discussions.

[1] L. Pitaevskii and S. Stringari, Bose-Einstein Condensation (Oxford University Press, Oxford, 2003).

[2] F. Dalfovo, S. Giorgini, L. Pitaevskii, and S. Stringari, Rev. Mod. Phys. 71, 463 (1999).

[3] C. J. Pethick and H. Smith, Bose-Einstein condensation in dilute gases (Cambridge University Press, Cambridge, 2001).

[4] A. J. Leggett, Rev. Mod. Phys. 73, 307 (2001).

[5] M. Olshanii, Phys. Rev. Lett. 81, 938 (1998).

[6] M. Girardeau, J. Math. Phys. 1, 516 (1960). 
[7] T. Kinoshita, T. Wenger, and D. S. Weiss, Science 305, 1125 (2004).

[8] B. Paredes et al., Nature 429, 277 (2004).

[9] L. S. Cederbaum and A. I. Streltsov, Phys. Lett. A 318, 564 (2003).

[10] O. E. Alon and L. S. Cederbaum, Phys. Rev. Lett. 95, 140402 (2005).

[11] Th. Busch, B. G. Englert, K. Rzazewski, and M. Wilkens, Found. Phys. 28, 549 (1998).

[12] M. A. Cirone, K. Góral, K. Rzazewski, and M. Wilkens, J. Phys. B 34, 4571 (2001).

[13] Y. Hao, Y. Zhang, J. Q. Liang, and S. Chen, cond-mat/0602483 (2006).

[14] K. Sakmann, A. I. Streltsov, O. E. Alon, and L. S. Cederbaum, Phys. Rev. A 72, 033613 (2005).

[15] Z. Idziaszek and T. Calarco, quant-ph/0604205 (2006).

[16] D. Blume and C. H. Greene, Phys. Rev. A 65, 043613 (2002).

[17] E. L. Bolda, E. Tiesinga, and P. S. Julienne, Phys. Rev. A 68, 032702 (2003).

[18] F. M. E. Tiesinga, C.J. Williams and P. Julienne, Phys. Rev. A 61, 063416 (2000).

[19] Z. Idziaszek and T. Calarco, Phys. Rev. A 71, 050701 (2005).

[20] D. Masiello, S. B. McKagan, and W. P. Reinhardt, Phys. Rev. A 063624 (2005).

[21] A. I. Streltsov, O. E. Alon, and L. S. Cederbaum, cond-mat/0603212 (2006).

[22] F. Deuretzbacher, K. Bongs, K. Sengstock, and D. Pfannkuche, cond-mat/0604673 (2006).

[23] D. Sundholm and T. Vänskä, J. Phys. B 37, 2933 (2004).

[24] T. Haugset and H. Haugerud, Phys. Rev. A 57, 3809 (1998).

[25] S. Klaiman, N. Moiseyev, and L. S. Cederbaum, Phys. Rev. A 73, 013622 (2006).

[26] H.-D. Meyer and G. A. Worth, Theor. Chem. Acc. 109, 251 (2003).

[27] H.-D. Meyer, in The Encyclopedia of Computational Chemistry, edited by P. v. R. Schleyer et al. (John Wiley and Sons, Chichester, 1998), Vol. 5, pp. 3011-3018.

[28] M. H. Beck, A. Jäckle, G. A. Worth, and H.-D. Meyer, Phys. Rep. 324, 1 (2000).

[29] S. Kuhr et al., Science 293, 278 (2001).

[30] B. Mohring et al., Phys. Rev. A 71, 053601 (2005).

[31] K. Huang and C. N. Yang, Phys. Rev. 105, 767 (1957).

[32] H.-D. Meyer, U. Manthe, and L. S. Cederbaum, Chem. Phys. Lett. 165, 73 (1990).

[33] O. Penrose and L. Onsager, Phys. Rev. 104, 576 (1956).

[34] G. A. Worth, M. H. Beck, A. Jäckle, and H.-D. Meyer, The MCTDH Package, Version 8.2, (2000). H.-D. Meyer, Version 8.3 (2002). See http://www.pci.uni- 
heidelberg.de/tc/usr/mctdh/.

[35] R. Kosloff and H. Tal-Ezer, Chem. Phys. Lett. 127, 223 (1986).

[36] T. Busch and G. Huyet, J. Phys. B 36, 2553 (2003).

[37] M. Girardeau, E. M. Wright, and J. M. Triscari, Phys. Rev. A 63, 033601 (2001).

[38] E. H. Lieb, R. Seiringer, and J. Yngvason, Phys. Rev. Lett. 91, 150401 (2003).

[39] G. Theocharis, P. Schmelcher, P. G. Kevrekidis, and D. J. Frantzeskakis, cond-mat/0505127 (2005). 